\title{
Angiology
}

The Journal of Vascular Diseases

\section{The Effect of Poststenotic Vessel Wall Compliance upon the Pulsus Tardus Phenomenon}

\author{
Ronald O. Bude, M.D. \\ Jonathan M. Rubin, M.D., Ph.D. \\ Joel F. Platt, M.D. \\ and Ronald S. Adler, M.D., Ph.D.
}

ANN ARBOR, MICHIGAN

\section{ABSTRACT}

Recent studies have investigated the detection of significant arterial stenoses through identification of the "pulsus tardus" phenomenon in Doppler waveforms obtained distal to the stenosis. The etiology of this phenomenon, however, has not yet been determined. Using an in vitro model based upon an electrical circuit analogy, the authors had as their objective to determine whether the compliance of the vessel wall immediately distal to a stenosis, in conjunction with the stenosis, is the cause of pulsus tardus. For a constant stenosis, it was found that the degree of pulsus tardus, as quantitated by the acceleration index, increased as the compliance of the poststenotic segment increased. It is concluded that pulsus tardus distal to an arterial stenosis is likely due to the compliance of the normally distensible artery, in conjunction with the stenosis. Pathological conditions that alter the compliance of the poststenotic segment may affect the degree of pulsus tardus, perhaps limiting its usefulness for upstream stenosis detection. 


\section{Introduction}

Recently, much attention has been devoted to the sonographic detection of arterial stenoses through evaluation of downstream Doppler waveforms. Studies have been conducted in the intracranial/carotid, ${ }^{1}$ renal, ${ }^{2-5}$ and penile arteries. ${ }^{6}$ These authors have used a parameter called "pulsus tardus" (defined as "a pulse beat slow to rise and fall"7), which is identified in downstream Doppler waveforms, to detect significant (greater than 50-80\%) upstream stenoses. Although hypotheses have been proposed as to the cause of pulsus tardus, ${ }^{1,3,4}$ the true etiology remains obscure.

To us, the pulsus tardus component of a poststenotic waveform was very reminiscent of the dampened signal produced by the passage of an alternating current through a low-pass filter (an RC [resistive-capacitive] circuit [Figure 1]). Since it is well known that pulsatile fluid flow and alternating current $(\mathrm{AC})$ circuits are often analogues, a fluid-flow analogue of an RC circuit (with the stenosis the analogue of the resistance and the compliance of the poststenotic vessel wall the analogue of the capacitance) was designed and tested in an effort to determine the etiology of pulsus tardus. If the etiology of pulsus tardus is known, it may be possible to predict conditions that limit its usefulness for stenosis detection.

\section{Methods and Materials}

The flow phantom for this series of experiments was constructed as illustrated (Figure 2). The ingress and egress tubes opened into a large plastic container that was partially filled with a fluid containing acoustic scatterers. Immersed within this fluid were a high-grade stenosis, lengths of tubing of variable compliance immediately distal to the stenosis, and all other tubing immediately proximal and distal to the stenosis. Scanning was performed in this fluid bath. All tube interconnections were made with plastic fittings, metallic screw clamps, and/or tape. All lengths of gum rubber tubing were of the same type, with an inside diameter (ID) of $0.42 \mathrm{~cm}$ and an outside diameter (OD) of $0.80 \mathrm{~cm}$.
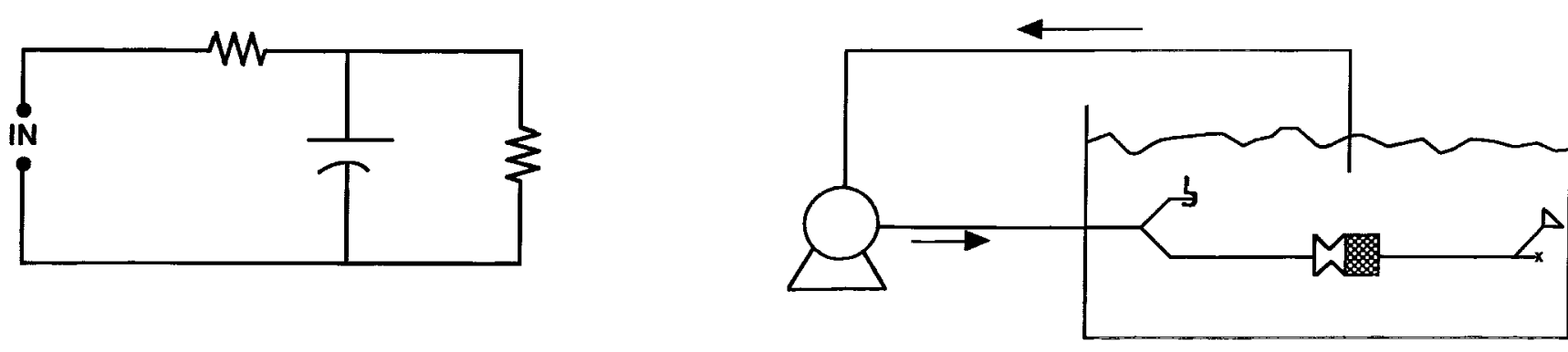

Figure 1. Schematic diagram of an RC circuit. The terminal labelled "IN" represents an alternating current power source. Regions with jagged lines represent resistors. The region consisting of a gap bounded by straight and curved lines represents a capacitor. At the right side of the diagram, the capacitor "short circuits" the higher frequency components to ground, while the $\mathrm{DC}$ and the lower frequency components pass through the resistor at the far right of the diagram.

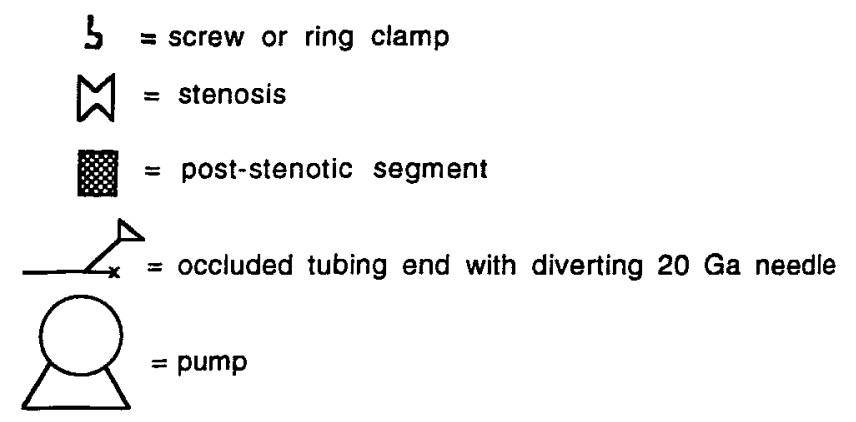

Figure 2. Schematic diagram of the experimental system (not drawn to scale). 
The pump assembly consisted of a pulsatile blood pump with plastic tubing $(0.95 \mathrm{~cm}$ ID and $1.4 \mathrm{~cm}$ OD) serving as an egress and an 18 French Foley catheter serving as an ingress. After a length of $90 \mathrm{~cm}$, the egress tubing was downsized to gum rubber tubing. After $60 \mathrm{~cm}$ of gum rubber tubing, a " $\mathrm{Y}$ " connection diverted the pump egress through two lengths of gum rubber tubing. One piece, approximately $6 \mathrm{~cm}$ in length, was partially clamped at its distal end and served as a shunt. This was necessary because some of the connections leaked if all of the pump output flowed through the length of tubing with the stenosis (to be described below), even at the lowest pump setting ( 5 cc per stroke). Once partially clamped, no leakage at tube interconnections occurred, and this portion of tubing remained unaltered throughout the experiment. The gum rubber tubing connected to the other arm of the $Y$ connector was approximately $60 \mathrm{~cm}$ in length and was connected to a stenosis at its distal aspect.

A stenosis was created from a $1 \mathrm{~cm}$ length of small copper tubing, of the kind used for fluid flow in small engines and other hobby applications. The stenotic segment had an ID of 0.079 $\mathrm{cm}$. It was wound with cellophane tape, which was subsequently trimmed to match the length of the stenotic segment, until a snug slide fit was achieved with the gum rubber tubing, attaining an $81 \%$ diameter stenosis with the $0.42 \mathrm{~cm}$ ID tubing. It was held in place with a metal screw clamp, which also secured the proximal portion of the balloon or syringe.

The lengths of tubing of variable compliance immediately distal to the stenosis consisted of a $4 \mathrm{~cm}$ length of a $3 \mathrm{cc}$ syringe (with the tapered end cut off) and 0.5 and $3.0 \mathrm{~cm}$ lengths of a rubber balloon of constant diameter. The OD of the gum rubber tubing matched the ID of the syringe, thus providing almost an exact slide fit. The balloon had an ID that was only minimally greater than the OD of the gum rubber tubing, and was nearly a slide fit. The syringe was essentially nondistensible and was presumed to have a low compliance. The balloon was presumed to have a larger compliance than the syringe. The use of two lengths of balloon allowed us to obtain two different compliances with the balloon, with the $3 \mathrm{~cm}$ length assumed to have approximately 6 times the compliance of the 0.5 $\mathrm{cm}$ segment $(3 \mathrm{~cm} / 0.5 \mathrm{~cm}=6)$. In this fashion, three different poststenotic compliances were produced, ranging from the low compliance of the nondistensible syringe to the relatively large compliance of the $3 \mathrm{~cm}$ length of distensible balloon. Since we were interested in the qualitative effects, we did not measure the absolute compliances of these segments.

The stenosis segment tubing assembly was completed with a $20 \mathrm{~cm}$ length of gum rubber tubing, attached at its proximal aspect to the lengths of tubing of variable compliance. A hemostat clamped the most distal aspect of this length of gum rubber tubing, with a 20 gauge needle inserted slightly proximal to the hemostat to simulate a resistance from a distal vascular bed.

The echogenic fluid medium consisted of 6.0 $\mathrm{g}$ of cornstarch mixed with 5.0 liters of room temperature water. The recirculating flow of the pump, augmented by sporadic hand stirring, kept the cornstarch adequately suspended within the water. Adequate Doppler waveforms were easily obtained from this mixture.

All experiments were performed with the following pump settings: (1) pump frequency of 60 cycles/minute; (2) duty cycle of 0.4 (duty cycle is the fraction of the entire cycle occupied by "systole"); and (3) pump volume of $5.0 \mathrm{cc}$ per cycle.

Sonographic studies were performed with an ultrasound unit, employing a sector-scanning transducer with a frequency of $10 \mathrm{MHz}$. Hardcopy images of the Doppler waveforms were recorded on film.

With the flow assembly set up as indicated (Figure 2), three different experiments were performed. During these experiments, the type of immediate poststenotic segment was varied with all other parameters held constant. The poststenotic segments consisted of the nondistensible syringe and the two different lengths of balloon as previously described. Doppler waveforms were obtained greater than 10 vessel diameters proximal to the stenotic segment, and also in the gum rubber tubing at least 10 vessel diameters distal to the anastomosis of the interchangeable poststenotic segment with the gum rubber tubing. These lengths insured that the pressures had stabilized within the flow streams. ${ }^{8}$

In order to quantitate the change in the degree of pulsus tardus, the acceleration indices (AI) of prestenotic and poststenotic waveforms were calculated according to previously published standards. ${ }^{2}$ For the sake of comparison, the AIs of the poststenotic waveforms were normalized relative to the prestenotic waveform. 


\section{Results}

The waveforms obtained with immediate poststenotic segments of varying degrees of compliance are as illustrated (Figure 3 ). It can be seen that as the degree of compliance of the poststenotic segment increases, the initial slope of the waveform decreases (the waveform is slower to rise). In other words, the degree of pulsus tardus increases as the degree of compliance of the poststenotic segment increases. Similarly, the AI (which should decrease with an increasing degree of pulsus tardus) decreased as the degree of compliance of the poststenotic segment increased (Figure 3).

\section{Discussion}

Recent studies have suggested that a detectable Doppler waveform alteration, which consists of a slowed rise to peak systole and has been termed "pulsus tardus" (a representative clinical example of pulsus tardus is illustrated in Figure 4), occurs distal to an arterial stenosis. ${ }^{1-6}$ This observation is important, for it may enable noninvasive identification of significant proximal stenoses through insonation of distal or parenchymal arteries, even though the stenosis itself is sonographically inaccessible (the kidney is a prime example, since the main renal artery cannot always be insonated). ${ }^{3,4}$ Attention has been directed to quantifying the degree of pulsus tardus, ${ }^{1-4}$ with the $\mathrm{AI}^{2}$ appearing to be the most promising of several developed indices.

The etiology of pulsus tardus, however, remains obscure. Owing to the many similarities between fluid flow systems and electrical circuits, it was conjectured the answer might be found by using an electrical circuit analogy. A tardus waveform, with its slow rise to peak velocity, is reminiscent of the alteration undergone by an alternating current after passage through a low-pass filter (ie, an RC circuit, Figure 1), which attenuates the high-frequency components of the waveform. In a pulsatile fluid flow system, a vessel with a distensible wall that expands during systole (analogous to the charging of a capacitor) and contracts during diastole (analogous to the discharging of a capacitor) located immediately distal to a stenosis was used to model an RC circuit. This model was considered to be appropriate, for the normal arterial wall pulsates in such a countercyclical fashion during the cardiac cycle.

Since the degree of pulsus tardus increased with increasing compliance of the poststenotic segment (Figure 1) in our experiments, it seems likely that production of pulsus tardus is dependent upon the degree of compliance of the poststenotic vessel wall. Since a normal artery is distensible, we believe our results show that pulsus tardus distal to an arterial stenosis is due to, and dependent upon, the compliance of the arterial wall in conjunction with the stenosis.

Based upon these experimental results, the possible conclusion is that significant poststenotic atherosclerotic disease may produce false-negative results when the presence of downstream pulsus tardus is used to detect a significant upstream stenosis. This is because a severely atherosclerotic artery would likely be rigid, perhaps rigid enough to be equivalent to our poststenotic segment of syringe. If this is the case, downstream pulsus tardus may not be observed, leading to a false-negative result in stenosis detection.

Before any firm conclusions are drawn from our results, a limitation of our experiment must be considered. Although our experimental design mimics the cardiovascular system, it does not possess exactly the same properties. For instance, the compliances of the gum rubber tubing and the balloon almost certainly do not match that of normal artery. Additionally, the viscosity of our cornstarch-in-water suspension was almost certainly different from that of blood. Therefore, although our results qualitatively show that changes in poststenotic vessel wall compliance can materially affect the pulsus tardus phenomenon and must be occurring to at least some degree in vivo, the degree to which they occur may be different in vivo from what was observed experimentally. Further in vivo study would be required to address this issue.

\section{Conclusion}

We have shown that the degree of pulsus tardus distal to a stenosis is dependent upon the degree of compliance of the poststenotic vessel wall in conjunction with the stenosis. It may prove to be the case that poststenotic atherosclerotic disease interferes with the pulsus tardus phenomenon enough to produce a false- 


\section{Figure 3.}

Doppler waveforms obtained during the experiment.

A. Representative Doppler waveform obtained proximal to the stenosis (the

prestenosis waveforms for all experiments were essentially indistinguishable). The AI's of all subsequent waveforms were normalized relative to this prestenosis waveform. B. Waveforms obtained with the syringe distal to the stenosis. The normalized AI was 0.90 . Although not unity, a normalized value of 0.90 is considered close enough to the prestenotic AI to indicate that there is no appreciable difference in the slopes of the systolic upsweeps of these two waveforms. This is because the initial slope of the systolic upsweep of both the prestenotic waveform and this waveform were so nearly vertical that measurement error was increased, making it likely that the observed difference, especially when compared to the poststenotic waveforms that follow, is due to measurement error.

C. Waveforms with a $0.5 \mathrm{~cm}$ length of balloon distal to the stenosis. Normalized AI was 0.37 .

D. Waveforms with a $3.0 \mathrm{~cm}$ length of balloon distal to the stenosis. There is almost no waveform pulsatility, and the AI was so low it could not be measured accurately.
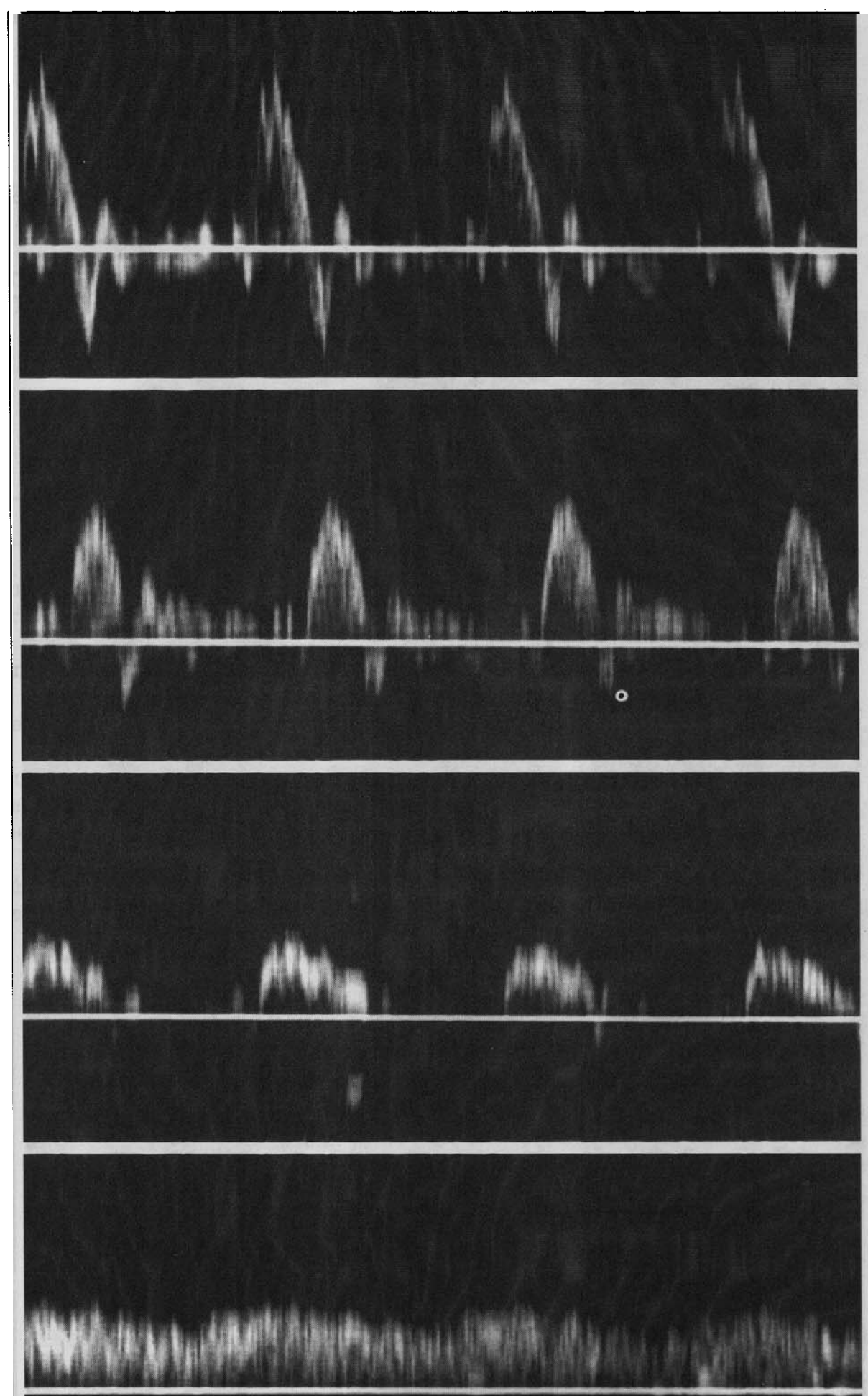

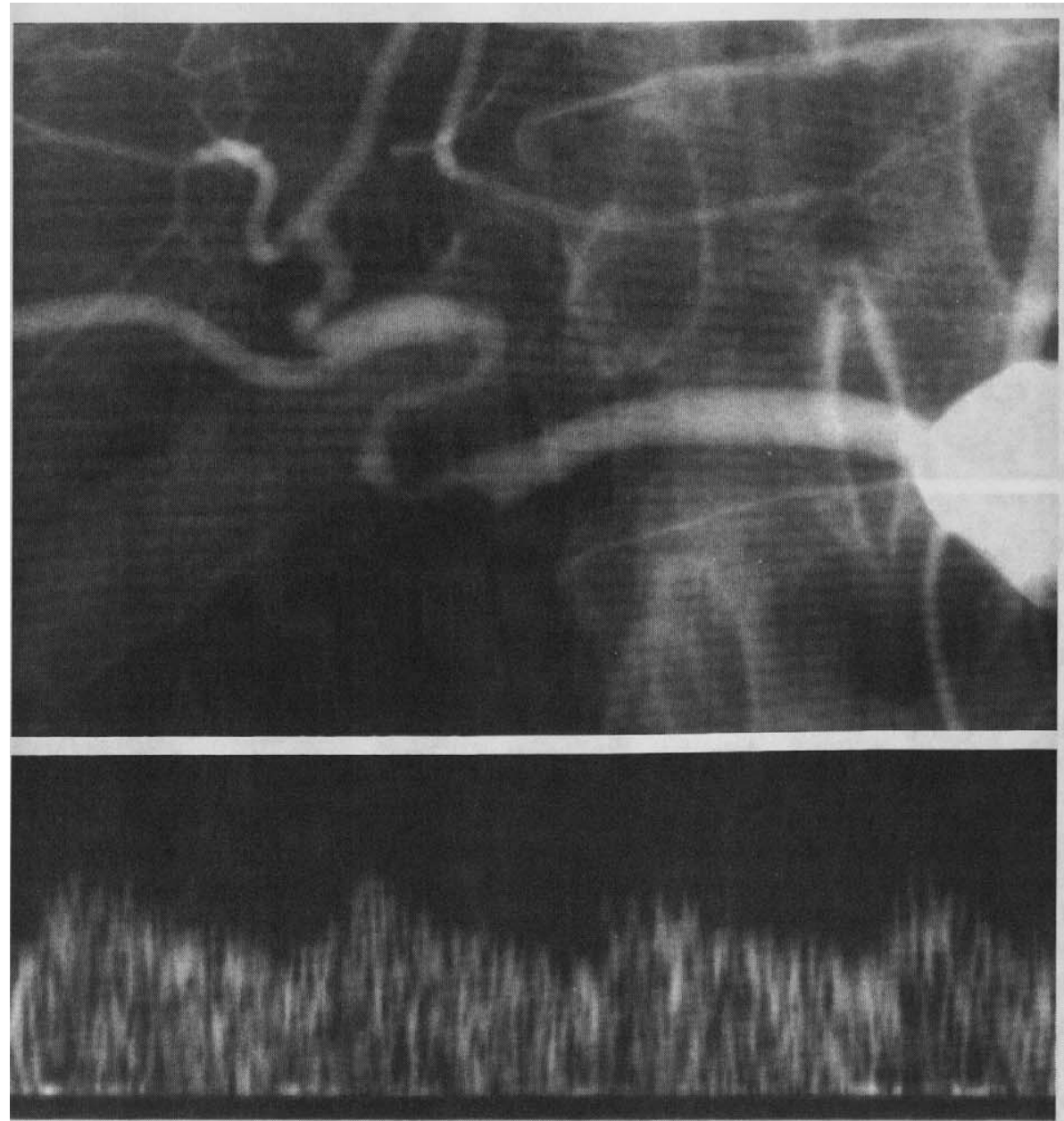

Figure 4.

Hepatic transplant artery stenosis.

A. Celiac arteriogram shows a long segment stenosis of the hepatic artery, with a maximum diameter stenosis of approximately $70 \%$.

B. Hepatic arterial Doppler waveform, obtained distal to the stenosis three days prior to angiography, showing a marked tardus component.

C. Comparison hepatic arterial waveform in a normal volunteer. Note the lack of a tardus component, and the distinct difference between this waveform and the poststenotic waveform of part $B$.
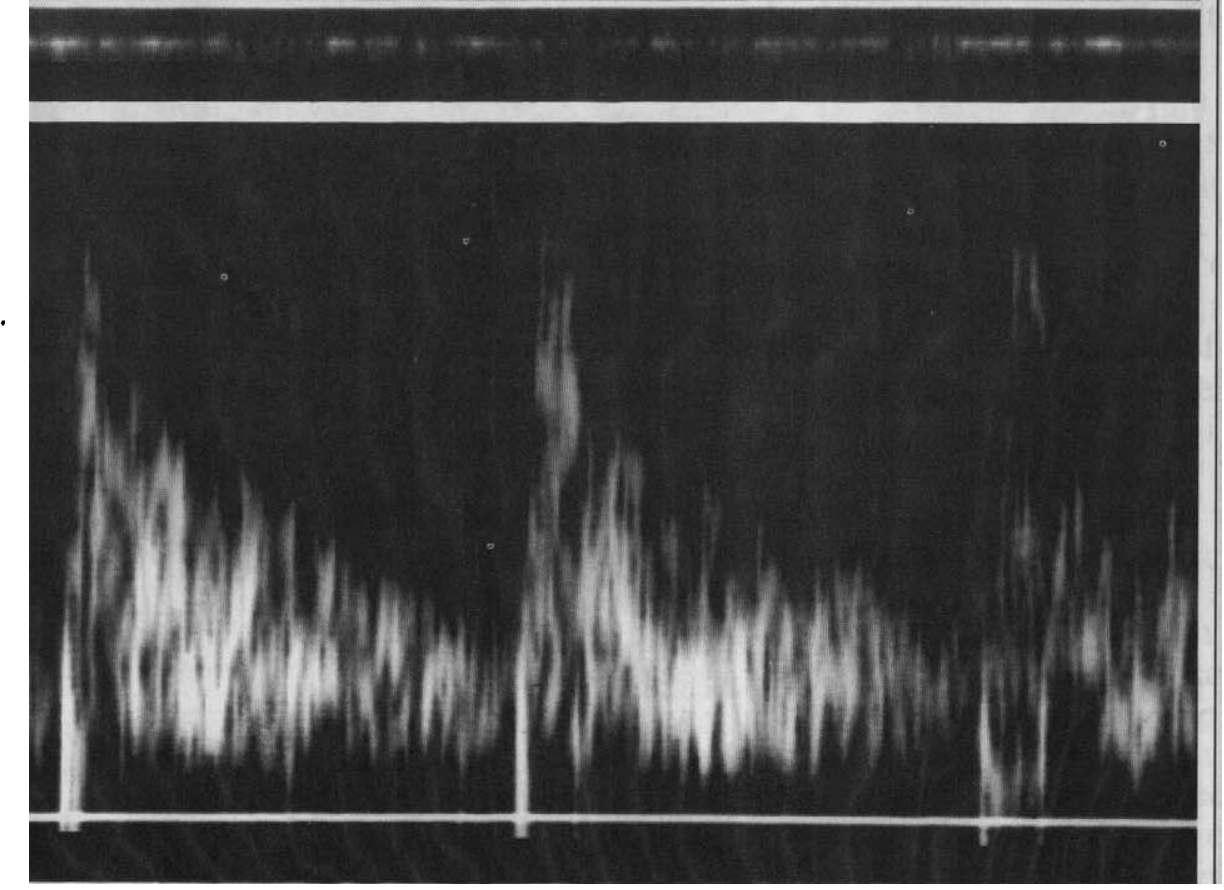
negative result when the distal pulsus tardus phenomenon is used to screen for significant upstream stenosis.

Ronald O. Bude, M.D.

Department of Radiology

University of Michigan Medical Center

Taubman Center 2910

1500 E. Medical Center Drive

Ann Arbor, MI 48109/0326

1. Kotval PS: Doppler waveform parvus and tardus: A sign of proximal flow obstruction. J Ultrasound Med 8:435-440, 1989.

2. Handa N, Fukunaga R, Etani H, et al: Efficacy of echo-Doppler examination for the evaluation of renovascular disease. Ultrasound in Med \& Biol 14:1-5, 1988.

3. Lafortune $M$, Patriquin $H$, Demeule E, et al: Renal arterial stenosis: Slowed systole in the downstream circulation-experimental study in dogs. Radiology 184:475-478, 1992.

4. Patriquin HB, Lafortune $M$, Jéquier JC, et al: Stenosis of the renal artery: Assessment of slowed systole in the downstream circulation with Doppler sonography. Radiology 184:479-485, 1992.

5. Memel DS, Dodd GD, Shah AN, et al: Imaging of en bloc renal transplants: Normal and abnormal postoperative findings. AJR 160:75-81, 1993.

6. Valji K, Bookstein JJ: Diagnosis of arteriogenic impotence: Efficacy of duplex sonography as a screening tool. AJR 160:65-69, 1993.

7. Asimov I, Bassett DL, Beamer PR, et al: Illustrated Stedman's Medical Dictionary, ed. 20. Baltimore: Williams \& Wilkins, 1961.

8. Huges WF, Brighton JA: Fluid Dynamics, Schaum's Outline. New York: McGraw-Hill, 1967, p 87. 